\title{
Quelques nouveautés concernant la flore vasculaire du Maroc septentrional : Jbel Tazzeka, Jbel Mezgout, région d'Al Hoceima
}

\author{
Mathieu Chambouleyron', Matthieu Bidat ${ }^{2}$, Matthieu Charrier ${ }^{3}$ \& Jean-François Léger ${ }^{1}$ \\ ${ }^{1}$ Emirates Center for Wildlife Propagation, Province de Boulemane, BP 47, 33250 Missour, Maroc. ${ }^{2} 380$ rue du Moulin \\ de Chareau, 45390 Puiseaux, France. ${ }^{3}$ Flora Consult, 13 bis avenue Louis Amouriq, 13290 Aix-en-Provence, France
}

\section{Correspondence}

M. Chambouleyron

e-mail: mchambouleyron@yahoo.fr

Recibido: 13 septiembre 2017

Aceptado: 25 noviembre 2017

Publicado on-line: décembre 2017
Some novelties about the vascular flora of northern Morocco: Jbel Tazzeka, Jbel Mezgout, Al Hoceima surroundings

Mots-clés: Flore vasculaire, inventaire, chorologie, Maroc.

Key words: Vascular flora, Inventory, Chorology, Morocco.
La présente note porte à connaissance des observations intéressantes issues de quatre excursions botaniques menées en 2011 dans le Maroc septentrional (fig. 1) : littoral méditerranéen de la région d'Al Hoceima (17.IV et 19-20.XI.2011), Jbel Mezgout dans le Rif oriental (21-22.V.2011) et Jbel Tazekka dans le Moyen-Atlas (9-10.IV.2011).

Les relevés botaniques réalisés lors de ces prospections ont révélé 12 taxons nouveaux ou confirmés pour un des secteurs biogéographiques du nord du Maroc, dont :

Un taxon nouveau pour l'Afrique et le Maroc : Orobanche santolinae Loscos \& J. Pardo (découvert dans le Rif oriental, sur le Jbel Mezgout) ;

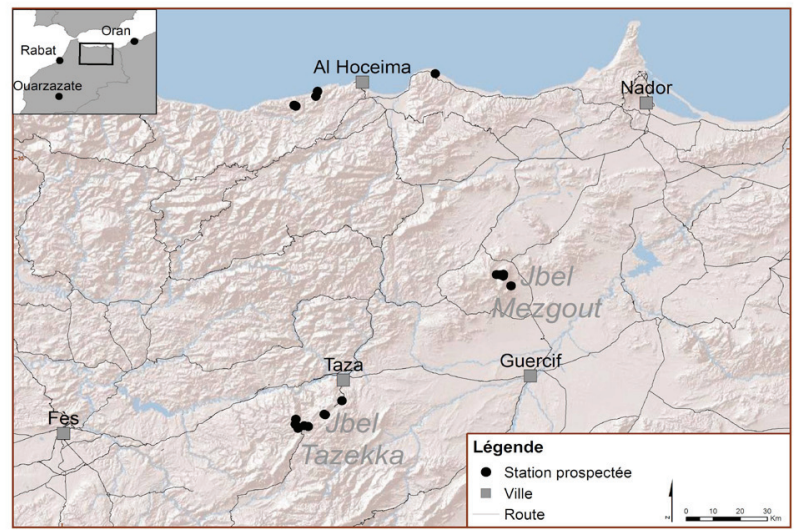

Figure 1. Localités prospectées. Figure 1. Surveyed localities
Un taxon nouveau pour le Littorral méditerranéen marocain : Narcissus viridiflorus Schousb. ;

Deux taxons nouveaux pour le Moyen-Atlas : Clypeola jonthlaspi subsp. microcarpa (Moris) Arcang. et Plantago serraria L..

Certaines de ces découvertes proviennent de secteurs pourtant relativement bien étudiés par le passé, tels que le Jbel Tazzeka ou le Littoral méditerranéen marocain (Dobignard, 2002).

\section{Observations}

Les résultats sont présentés par région naturelle selon les découpages biogéographiques proposés par Fennane \& IbnTattou (2005) et IbnTattou \& Fennane (2009).

La nomenclature adoptée dans cet article est conforme à celle de l'Index synonymique de la flore d'Afrique du Nord (Dobignard \& Chatelain, 20102013).

\section{Littoral méditerranéen}

\section{ANGIOSPERMES DICOTYLEDONES}

Amaranthaceae

Halogeton sativus Moq.

Al Hoceima (El RâsTârf) (N35.27547, W3.67150), 20m, rochers littoraux, 20.XI.2011, $M$. Bidat et M. Chambouleyron «observation».

Confirmation de la présence de ce taxon dans 
LM-1, dont le signalement à l'embouchure de l'oued Nekkor (LM-1) par Bendaanoun (1991: 555) n'a été repris ni par Fennane et IbnTattou (2005), ni par Romo (in Valdés et al., 2002).

\section{Boraginaceae}

Lithospermum arvense $\mathrm{L}$.

Adouz (Bokkoyas) (N35.16989, W4.16158), $490 \mathrm{~m}$, culture céréalière extensive,17.IV.2011, M. Bidat et M. Chambouleyron «observation».

Nouveau pour LM-1.

\section{ANGIOSPERMES MONOTYLEDONES}

\section{Amaryllidaceae}

Narcissus viridiflorus Schousb.

Taussart (Bokkoyas) (N35.20200, W4.09019), 340m, matorral, 19.XI.2011, M. Bidat et $M$. Chambouleyron ( $\mathrm{Hb}$. privé Chambouleyron).

Nouveau pour LM (ici LM-1). Cette nouvelle station étend l'aire de distribution connue de cette espèce d'une centaine de kilomètres vers l'est et marque sa limite orientale, puisqu'elle n'était jusque là connue que du sud de l'Andalousie (Valdés et al., 1987), de la péninsule tingitane et de la façade atlantique nord du Maroc (Fennane et al., 2014; IbnTattou \& Fennane, 2008).

\section{Orchidaceae}

\section{Serapias parviflora Parl. subsp. parviflora}

Adouz (Bokkoyas) (N35.16989, W4.16158), 490m, talus, 17.IV.2011, M. BidatetM. Chambouleyron (Hb. privé Chambouleyron).

Confirmé pour LM-1, où il était à rechercher selon IbnTattou \& Fennane (2008).

\section{Rif oriental : Jbel Mezgout}

\section{ANGIOSPERMES DICOTYLEDONES}

\section{Caryophyllaceae}

Minuartia stereoneura Mattf.

Saka (N34.57019, W3.44392), 1560m, rocher, 22.V.2011, M. Bidat et M. Chambouleyron ( $\mathrm{Hb}$. privé Chambouleyron).

La plante récoltée présente une pubescence atypique, avec des sépales à poils glanduleux sur tout le dos, atteignant presque leur extrémité (fig. 2). Maire (1963) indique certaines variétés de ce taxon à sépales présentant une légère pubescence glanduleuse, mais celle-ci limitée à leur base. Cette récolte ne présente pas d'autre particularité morphologique (pédicelles dépassant les bractées, sépales marqués d'une seule nervure saillante, fleurs à 10 étamines).

Ce taxon a déjà été découvert sur le Jbel

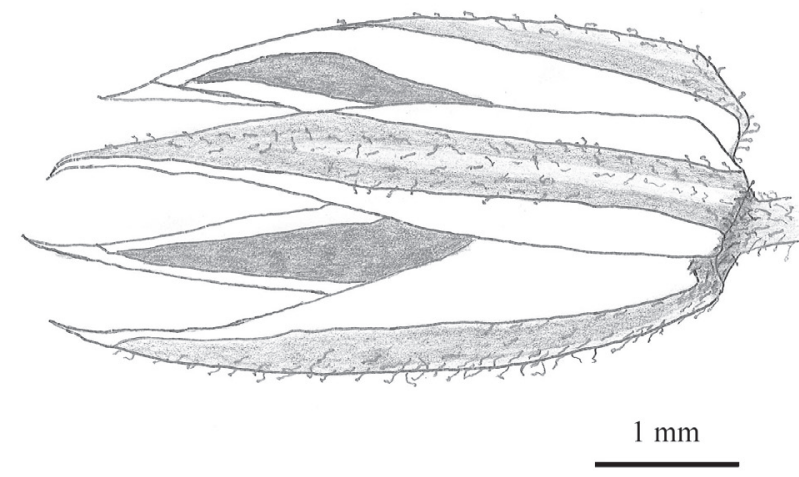

Figure 2. Calice de Minuartia stereoneura récoltée sur le Jbel Mezgout. Figure 2. Calyx of Minuartia stereoneura collected on the Jbel Mezgout.

Mezgout en 2004 par Molero Briones et Montserrat Marti (2006).

\section{Fabaceae}

Vicia lecomtei Humbert \& Maire

Saka (N34.57386, W3.45486), 1190 m, matorral, 22.V.2011, M. Bidat \& M. Chambouleyron (Hb. privé Chambouleyron).

Nouveau pour R-3. Ce taxon présente localement des gousses contenant généralement 4 graines et des inflorescences à 6-8 fleurs, ce qui correspond davantage à la diagnose proposée par Dobignard (2009) qu'à celles de Raynaud (1976) et de Hormat (in Fennane et al., 2007), ces derniers l'indiquant à gousse contenant 1-3 graines et à inflorescence pauciflore (3-4 fleurs).

\section{Linaceae}

Linum narbonense $\mathrm{L}$.

Saka (N34.57019, W3.44392), 1560m, rocher, 22.V.2011, M. Bidat et M. Chambouleyron ( $\mathrm{Hb}$. privé Chambouleyron) et ( $\mathrm{Hb}$ ECWP, leg. M. Bidat).

Nouveau pour R-3.

Orobanchaceae

Orobanche santolinae Loscos \& J. Pardo

Saka (N34.57481, W3.46642), 1030m, matorral, 22.V.2011, M. Bidat et M. Chambouleyron (Hb. privé Chambouleyron). Détermination Luis Carlón et Antonio José Pujadas-Salvà.

Nous renvoyons le lecteur au travail de Pujadas-Salvà (2010) pour ce qui est de la valeur taxonomique, parfois contestée, de ce taxon.

Nouveau pour l'Afrique et pour le Maroc (ici R-3). Jusqu'à présent, l'aire de distribution connue de ce taxon allait du sud-ouest de la France au sud de l'Espagne, lles Baléares incluses (PujadasSalvà, loc. cit.), mais sa découverte en Afrique du Nord était probable selon Pujadas-Salvà (loc. cit.).

Nous donnons ici la diagnose de notre 


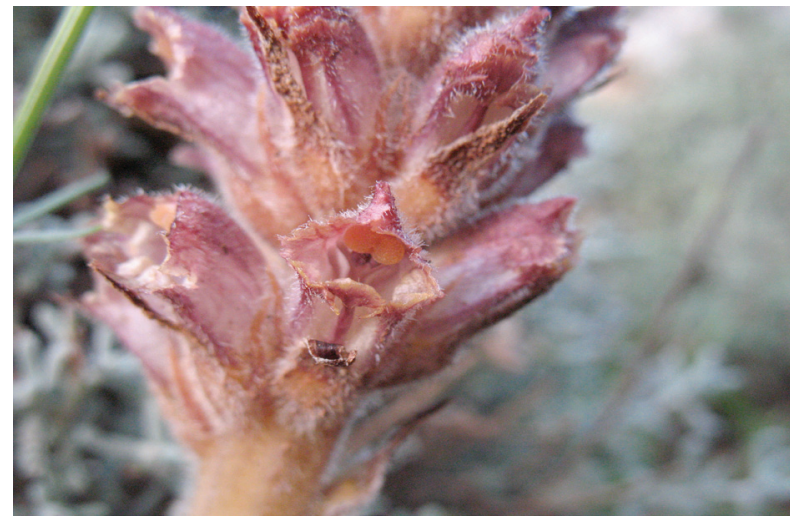

Figure 3. Corolle d'Orobanche santolinae (Jbel Mezgout). Figure 3. Corolla of Orobanche santolinae (Jbel Mezgout).

récolte (mesures faites sur la planche d'herbier, contenant une tige avec son inflorescence), et pour comparaison nous précisons entre crochets les valeurs indiquées par Pujadas-Salvà (loc. cit.) sur les récoltes ibériques qu'il a étudié :

Feuilles supérieures $11-13 \times 3,5 \mathrm{~mm}$ [(9)12-20x(3)4-8] lancéolées, dressées, velues glanduleuses. Inflorescence (en début d'épanouissement) $5,5-6 \times 2,5 \mathrm{~cm}[8-17 \times(2,1) 2,4-$ $3,2(3,9)]$. Bractées 10,5-13x3-3,5 mm [(8)1017(19)x4-7]. Calice 7,5-8 mm [8-14] à dents étroites, villeux-glanduleux. Corolle 14-15 mm [1518(20)], dressée-étalée, tubuleuse, droite, velueglanduleuse, de couleur blanche-pourpre (fig. 3). Lèvre supérieure émarginée, poilue-glanduleuse, lèvre inférieure à lobes ovales sub-égaux (le central très légèrement plus grand), poilue-glanduleuse. Filets staminaux $9 \mathrm{~mm}$ long, les adaxiaux insérés à $2,3 \mathrm{~mm}$ de la base de la corolle [(2)3-4(5)], les abaxiaux à 1,5-2 mm [2-3(4)], à poils simples abondants $(0,5 \mathrm{~mm}$ long) sur les $2 / 3$ inférieurs et poils glanduleux courts épars sur le $1 / 3$ supérieur. Anthère $1,5 \mathrm{~mm}[1,5-2]$, marron, avec quelques poils sur la partie inférieure de la suture. Ovaire poilu-glanduleux sur la partie supérieure. Style poilu-glanduleux. Stigmate bilobé orange.

Cette récolte rentre donc dans la plage de variation morphologique des plantes ibériques. L'inflorescence est un peu courte mais notre plante n'était pas totalement épanouie lors de la récolte, laissant présager son allongement. A noter toutefois un stigmate de couleur orange (fig. 3) alors qu'il est indiqué pourpre ou rarement blanc par Pujadas-Salvà (loc. cit.).

En Europe, ce taxon parasite diverses espèces appartenant au genre Santolina Tourn. ex L. (Pujadas-Salvà, loc. cit.). Sur le Jbel Mezgout, il parasite Santolina ascensionis Sennen ex Maire

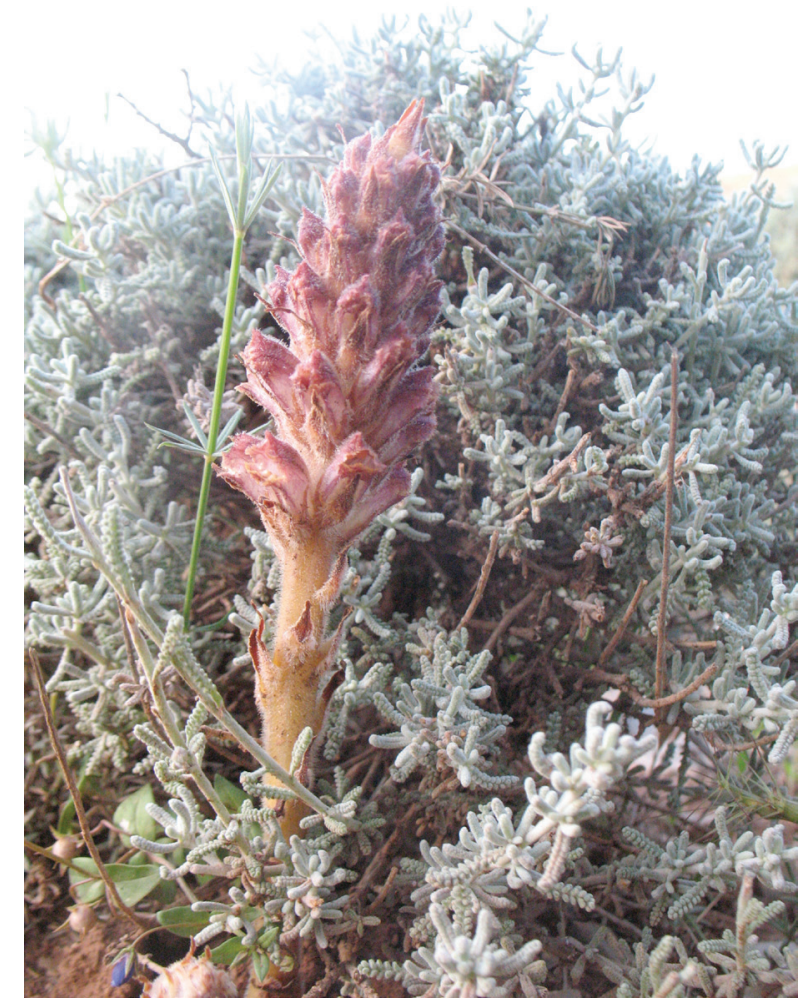

Figure 4. Orobanche santolinae parasitant Santolina ascensionis (Jbel Mezgout). Figure 4. Orobanche santolinae parasitizing Santolina adscensionis (Jbel

(fig. 4), taxon inclus dans S. africana Jord. \& Fourr. par Dobignard \& Chatelain (2011), hôte jusque-là inconnu.

\section{Moyen-Atlas : Jbel Tazekka}

\section{ANGIOSPERMES DICOTYLEDONES}

\section{Brassicaceae}

Clypeola jonthlaspi subsp. microcarpa (Moris) Arcang.

Bab Bou Idir (N34.05614, W4.13383), $1610 \mathrm{~m}$, pelouse, 09.IV.2011, M. Bidat, $M$. Chambouleyron, M. Charrier et J.-F. Léger ( $\mathrm{Hb}$. privé Chambouleyron).

Nouveau pour le Moyen-Atlas (ici MA-1). Ce taxon est signalé non loin, dans le Rif (Fennane \& IbnTattou, 2005 ; Montserrat in Valdés et al., 2002) et dans la région d'Aknoul (Montserrat in Valdés et al., 2002).

La valeur taxonomique des sous-espèces de Clypeola jonthlaspi L. est mise en doute par Tison \& De Foucault (2014).

Sisymbrella aspera subsp. munbyana (Boiss. \& Reut.) Greuter \& Burdet 
Bab Bou Idir (N34.09678, W4.07539), $1370 \mathrm{~m}$, bord d'étang, 09.04.2011, M. Bidat, M. Chambouleyron, M. Charrier et J.-F. Léger (Hb. privé Chambouleyron).

L'espèce sensu lato est cité de l'ensemble du Moyen-Atlas par Fennane \& IbnTattou (2005), mais cette sous-espèce n'avait pas encore été indiquée dans MA1. Spécimens immatures, mais sur lesquels on peut cependant distinguer l'épaisseur caractéristique du style des siliques. Pour la détermination infraspécifique, nous nous basons sur les tiges lisses et la longueur des fleurs $(7-9 \mathrm{~mm})$.

\section{Caryophyllaceae}

\section{Silene colorata subsp. trichocalycina} (Fenzl) Maire

Taza

(N34.14386, W4.01372), $1100 \mathrm{~m}$, matorral, 10.IV.2011, M. Bidat, $M$. Chambouleyron, M. Charrier et J.-F. Léger (Hb. privé Chambouleyron).

L'espèce sensu latoest citée de l'ensemble du Maroc, mais Fennane \& IbnTattou (2005) soulignent le manque de données pour la répartition des sous-espèces. Cette station est attribuée à MA-1.

\section{Cistaceae}

Helianthemum ruficomum Spreng.

Bab Bou Idir (N34.06383, W4.17836), 1700m, matorral, 10.IV.2011, M. Bidat, M. Chambouleyron, M. Charrier et J.-F. Léger ( $\mathrm{Hb}$. ECWP, leg. M. Chambouleyron).

Nouveau pour MA-1.

\section{Plantaginaceae}

\section{Plantago serraria L.}

Bab Bou Idir (N34.14386, W4.01372), 1100m, matorral, 10.IV.2011, M. Bidat, M. Chambouleyron, M. CharrieretJ.-F. Léger(Hb. Privé Chambouleyron) et ( $\mathrm{Hb}$. ECWP, leg. J.-F. Léger).

Nouveau pour le Moyen-Atlas (ici MA-1). L'espèce est connue à proximité, notamment dans la Basse Moulouya, le Rif et le Maroc atlantique nord (Fennane \& IbnTattou, 2005).

\section{Rosaceae}

Aphanes maroccana Hylander \& Rothm.

Bab Bou Idir (N34.05039, W4.16853), $1510 \mathrm{~m}$, chênaie, 10.IV.2011, M. Bidat, $M$. Chambouleyron, M. Charrier et J.-F. Léger ( $\mathrm{Hb}$. privé Chambouleyron).

Nouveau pour MA-1.

\section{ANGIOSPERMES MONOTYLEDONES}

\section{Orchidaceae}

Orchis italica Poir.

Bab Bou Idir (N34.05614, W4.13383), $1610 \mathrm{~m}$, yeuseraie, 09.IV.2011, M. Bidat, $M$. Chambouleyron, M. Charrier et J.-F. Léger ( $\mathrm{Hb}$. privé Chambouleyron).

Nouveau pour MA-1.

\section{Remerciements}

Nous sommes reconnaissants envers I'Emirates Center for Wildlife Propagation (ECWP) et I'International Fund for Houbara Conservation (IFHC) pour la mise à disposition de leurs ressources (matériel, fonds bibliographique et herbier). Nous adressons également nos vifs remerciements à MM. Luis Carlón (Jardín Botánico Atlántico de Gijón, Espagne) et Antonio José Pujadas-Salvà (Universidad de Córdoba, Espagne) pour leur aide à la détermination d'Orobanche santolinae.

\section{Références}

Bendaanoun, M. (1991). Contribution à l'étude écologique de la végétation halophile, halohygrophile et hygrophyle des estuaires, lagunes, deltas et sebkhas du littoral atlantique et méditerranéen et du domaine continental du Maroc (thèse de doctorat non publiée). Faculté des Sciences et Techniques de St Jérôme, Marseille, France.

Dobignard, A. (2002). Contributions à la connaissance de la flore du Maroc et de l'Afrique du Nord. Nouvelle série. 1. Journal de Botanique, 20, 5-43.

Dobignard, A. (2009). Contributions à la connaissance de la flore du Maroc et de l'Afrique du Nord. Nouvelle série. 2. Journal de Botanique, 46-47, 3-136.

Dobignard, A. \& Chatelain, C. (2010-2013). Index synonymique de la flore d'Afrique du Nord. 5 volumes. Conservatoire et Jardin botaniques de la Ville de Genève, ECWP, Genève.

Fennane, M. \& Ibn Tattou, M. (2005). Flore vasculaire du Maroc, inventaire et chorologie, Pteridophyta, Gymnospermae, Angiospermae p.p., volume 1. Travaux de l'Institut Scientifique Rabat, Série Botanique 37.

Fennane, M., Ibn Tattou, M., Ouyahya, A. \& El Oualidi, J. (dir.) (2007). Flore pratique du Maroc, Angiospermae (Leguminosae - Lentibulariaceae), volume 2. Travaux de l'Institut Scientifique Rabat, Série Botanique 38.

Fennane, M., Ibn Tattou, M. \& El Oualidi, J. (dir.) (2014). Flore pratique du Maroc, Dicotylédones (p.p.), Monocotylédones, volume 3. Travaux de I'Institut Scientifique Rabat, Série Botanique 40.

Ibn Tattou, M. \& Fennane, M. (2009). Flore vasculaire du Maroc, inventaire et chorologie, Asteraceae et Monocotylédones, volume 2. Travaux de l'Institut 
Scientifique Rabat, Série Botanique 39.

Maire, R. (1952-1987). Flore de l'Afrique du Nord. Lechevalier, Paris.

Molero Briones, J. \& Montserrat Marti, J.M. (2006). Novedades corológicas y taxonómicas selectas para el catálogo de la flora vascular del norte de Marruecos. Lagascalia, 26, 83-110.

Pujadas-Salvà, A.J. (2010). Typification and characterization of Orobanche santolinae Loscos \& J. Pardo (Orobanchaceae). Taxon, 59(3), 959-964.

Raynaud, C. (1976). Monographie et iconographie du genre Vicia L. au Maroc. Bulletin de l'Institut Scientifique, Rabat, 1, 147-172.

Tison, J.-M. \& De Foucault, B. (dir.) (2014). Flora Gallica. Flore de France. Biotope, Mèze.

Valdés, B., Talavera, S. \& Fernandez-Galiano, E. (dir.) (1987). Flora vascular de Andalucía Occidental. Ketres Editora S.A., Barcelona.

Valdés, B., Rejdali, M., Achhal El Kadmiri, A., Jury, J.L. \&Montserrat, J.M. (dir.) (2002). Catalogue des plantes vasculaires du Nord du Maroc, incluant des clés d'identification. CSIC, Madrid. 
